\title{
Ultrafast laser writing of optical waveguides in ceramic Yb:YAG: a study of thermal and non-thermal regimes
}

\author{
A. Benayas - W.F. Silva - A. Ródenas • C. Jacinto · J. Vázquez de Aldana - F. Chen • \\ Y. Tan · R.R. Thomsom - N.D. Psaila - D.T. Reid • G.A. Torchia · A.K. Kar • D. Jaque
}

Received: 9 August 2010 / Accepted: 12 November 2010 / Published online: 30 November 2010

(c) Springer-Verlag 2010

\begin{abstract}
We report the improvement of ultrafast laser written optical waveguides in Yb:YAG ceramics by tailoring the presence of heat accumulation effects. From a combination of ytterbium micro-luminescence and micro-Raman structural analysis, maps of lattice defects and stress fields have been obtained. We show how laser annealing can strongly reduce the concentration of defects and also reduce compressive stress, leading to an effective $50 \%$ reduction in the propagation losses and to more extended and symmetric propagation modes.
\end{abstract}

A. Benayas and W.F. Silva contributed equally to this work.

A. Benayas $(\varangle) \cdot$ D. Jaque

Departamento de Física de Materiales, Universidad Autónoma de Madrid, 28049 Madrid, Spain

e-mail: antonio.benayas@uam.es

W.F. Silva $\cdot$ C. Jacinto

Grupo de Fotônica e Fluidos Complexos, Instituto de Física, Universidade Federal de Alagoas, 57072-970 Maceió, Alagoas, Brazil

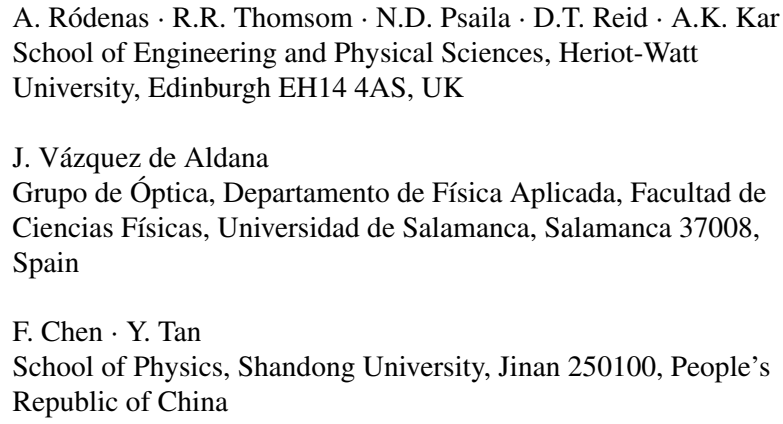

G.A. Torchia

Centro de Investigaciones Ópticas, CONICET-CIC,

1900 La Plata, Argentina

\section{Introduction}

Ultrafast Direct Laser Writing (DLW) has already been established itself as powerful technique for the fabrication of integrated photonic devices in dielectric media through the controlled modification of refractive index at the microscale. When compared to other microstructuring techniques, DLW shows several advantages such as truly threedimensional (3D) fabrication capability, absence of restrictive sample preparation requirements, a mainly one-step process, and suitability for processing large areas, short processing times, good repeatability and low manufacturing costs. DLW has been demonstrated to be a very powerful technique capable of producing structural changes and modifying the refractive index in transparent media. Indeed, DLW of waveguides (WGs), photonic crystals, microresonators and micro-diffraction elements has been already demonstrated in a great variety of dielectric materials including glasses and crystals [1-10]. These DLW devices have shown excellent properties, especially when referring to waveguides. DLW allows for the fabrication of buried channel waveguides in a wide range of optical materials whose propagation properties can be easily tailored by an adequate choice of the writing parameters [11-14]. Under appropriate writing conditions, WGs with low losses $(<0.6 \mathrm{~dB} / \mathrm{cm})$ and preserving the original properties of the inscribed material (electro-optical coefficients, laser gain, nonlinear response, Raman gain) have been already reported [15-21].

Among the different writing parameters that can be used for the WGs optimization, the repetition rate of femtosecond laser pulse train is one of the most critical parameters, as it affects the excitation-relaxation cycle between subsequent femtosecond laser pulses arrive at the focal spot volume. It has been previously demonstrated that when high repetition 
rates are used, thermal accumulation between consecutive pulses can greatly increase the temperature and drive fast annealing process at the moving laser focal volume [2225]. It has subsequently been postulated that the presence of local annealing effects could substantially affect the magnitude, extension and nature of the microstructural changes produced by DLW. In some materials, such as lithium niobate crystals, it has been shown how the use of high repetition rates leads to a reduction in the lattice damage, where the rapid annealing induced defect recombination was stated to be at the basis of the achieved superior optical performance (in terms of propagation loss) [15]. However, in such studies not only high repetition rates were used, but also different pulse overlapping, heat accumulation parameters, and laser pulse energies, therefore mixing different laser-crystal interaction effects on the same process. Here, we present a comprehensive study of structures inscribed using approximately the same parameters, except for the time between pulses which is varied almost three orders of magnitude (from $1 \mathrm{~ms}$ to $2 \mu \mathrm{s}$ ) to study its effect on the final device. To characterize the different channel waveguides resulting in each case, we present a detailed micro-spectroscopy analysis of the resultant crystal lattice changes together with comparison of the resultant waveguide guiding properties.

The crossover from the non-thermal to the thermal regime (in which the time between pulses matches the heat diffusion time from the focal volume) is determined by the critical frequency [24]:

$f_{c r}=\frac{D_{t h}}{d_{\text {laser }}^{2}}$

where $D_{t h}$ is the thermal diffusivity of the irradiated material and $d_{\text {laser }}$ is the focal spot diameter. Therefore, a normalized frequency parameter, $f_{n}=f_{\text {laser }} / f_{c r}$, is used to easily determine whether the waveguide is fabricated in presence or absence of thermal accumulation between consecutive pulses. It is important to note here that when the time between pulses is much longer than the heat diffusion time from the focal spot $(\sim 0.1-10 \mu \mathrm{s})$, rapid cooling takes place and the extension of the heat affected zone outside the focal volume is therefore completely negligible, and that regime can be classified as the non-thermal one, with $f_{n} \sim 0$. Once the pulse repetition rate is increased, so that no cooling can be effectively produced, the temperature at the focal volume will increase instead of decrease, and cooling will only be produced once the laser has moved to a different region of the sample. This writing regime is therefore regarded as the thermal one, with $f_{n} \geq 1$.

Among the different materials in which the writing of photonic circuits is desired, YAG polycrystalline ceramics (cYAG) are of particular relevance [26]. On one side, YAG ceramics constitute an excellent platform for the development of highly efficient laser circuits because their outstand- ing properties such as good mechanical and thermal properties and the possibility of large scale and composite fabrication. Besides, YAG ceramics can also be easily doped with high concentrations of rare-earth ions so that they offer a solid-state laser medium capable of low lasing threshold and high power laser generation. DLW has been already demonstrated to be a powerful technique for the fabrication of photonic crystals and waveguides in YAG ceramics, but only in the low repetition rate regime (1 kHz) [20, 27-29].

Fundamental studies have concluded that when a low repetition rate train of ultrashort pulses is tightly focused inside a YAG ceramic, local damage is produced at focus, and that this damage is accompanied by a lattice compression in its surroundings [30]. The first effect (damage) causes a refractive index reduction whereas the second effect (compression) leads to a local refractive index enhancement. The writing parameters and geometry strongly determine the dominant effect and, hence, the waveguiding mechanism. In this sense, it is possible to find in the literature both "stressinduced" and "damage-induced" YAG ceramic waveguides with completely different properties (the former are low loss waveguides whereas the latter shows a superior thermal stability) [31]. The "double filament" approach, which uses both mechanisms (stress and damage) in a complementary way, has been found to be especially suitable for the fabrication of waveguides in YAG with tailored properties. Doping with neodymium ions provides the fabricated WGs with optical gain that has been used in the past for highly efficient laser light generation [20]. Nevertheless, all the structures fabricated up to now have been written with low repetition rates, and the potential benefit of using high repetition rate laser sources as a way of improving the waveguide quality is still unexplored. In addition, no attempt to fabricate these WGs in YAG ceramics doped with other laser ions different from $\mathrm{Nd}^{3+}$ has been performed, to the best of our knowledge.

Ytterbium doped YAG ceramics (Yb:cYAG) have demonstrated in the past an outstanding laser performance in both continuous wave and pulsed regimes [32,33]. When compared to $\mathrm{Nd}^{3+}$ ions, ytterbium doping shows different advantages most of them based on their very simple energy level diagram. This avoids excited state absorption of laser and pump radiations and leads to low quantum defect between pump and laser photons (thus reducing thermal loading during laser operation). In addition, $\mathrm{Yb}^{3+}$ ions exhibit a strong photon-phonon coupling [34]. This weak isolation from the environment therefore makes ytterbium ions excellent luminescence probes for the detection of microstructural changes that can be induced in the YAG ceramic network. This characteristic feature can be used, for instance, to determine how the YAG network is modified by ultrashort pulses in the presence or absence of heat accumulation between consecutive pulses. Despite its interest from both an 
applied and fundamental point of view, and also despite the outstanding results recently obtained from DLW fabricated Yb:YAG crystalline waveguides [35], there are neither reports on the DLW of waveguides in Yb:YAG ceramics in the thermal and non-thermal regimes, nor any attempt to elucidate the nature and extension of the microstructural changes induced in the YAG network.

In this work we report on the waveguides fabricated by DLW in Yb:YAG ceramic in the thermal and nonthermal regimes by using high $(500 \mathrm{kHz})$ and low $(1 \mathrm{kHz})$ writing frequencies. The optical properties (propagation modes, polarization dependences and propagation losses) of waveguides fabricated under the same pulse energy, fluence, and pulse overlapping conditions were systematically compared. The microstructural modifications induced in the YAG network in each case were investigated by confocal fluorescence and Raman microscopy mapping experiments. The differences observed in the structural properties of the structures fabricated in presence/absence of thermal accumulation effects have been discussed and related to the different propagation properties of the structures, leading to an effective improvement in waveguide properties with respect to previous works.

\section{Experimental}

\subsection{Waveguide writing}

The Yb:cYAG sample used in this work was a $2 \times 10 \times 6 \mathrm{~mm}$ sample provided by Baikowski Inc. The nominal $\mathrm{Yb}^{3+}$ concentration was 1 at.\%, and the average grain size was determined by scanning electron microscopy (SEM) experiments to be close to $2 \mu \mathrm{m}$. For the purpose of WG laser writing all the samples' faces were polished to optical quality. Two sets of WGs were written $400 \mu \mathrm{m}$ below the surface. In both sets we used the transverse geometry in which the infrared pulses were focused inside the sample through the $10 \times 6 \mathrm{~mm}$ face and the sample was translated parallel to the $10 \mathrm{~mm}$ dimension. WG were fabricated by the consecutive writing of two parallel damage tracks separated by $20 \mu \mathrm{m}$ (i.e. a "doubleline" approach). Both the $400 \mu \mathrm{m}$ writing depth and $20 \mu \mathrm{m}$ separation between tracks are known to be parameters which yield good single mode confinement around $1 \mu \mathrm{m}$ wavelength and therefore these are taken as standard. The separation between pulses along the scan direction was kept constant to about $4-5 \mathrm{~nm}$, yielding a constant overlapping rate in the sample of about $99.8 \%$ (thus ensuring same total fluence dose). The laser polarization and focusing optics were also the same. As two lasers with different repetition rates were employed, different translation speeds had to be used, in order to keep the pulse overlapping rate to the same value. The two different experimental setups used are the following: (a) Thermal regime: $\mathrm{A} \mathrm{Yb}$ doped fibre laser (Fianium Ltd.) providing $\approx 300 \mathrm{fs}$ pulses at $\lambda_{0}=1064 \mathrm{~nm}$ with a pulse repetition rate of $500 \mathrm{kHz}$. The laser polarization was adjusted to be perpendicular to both the incident beam and sample translation direction. The writing beam was focussed into the Yb:cYAG sample by using an aspheric 0.4 numerical aperture lens, giving a spot diameter of $1.22 \times \lambda_{0} / \mathrm{NA}=3.2 \mu \mathrm{m}$. (That calculation did not take into account the fact that we were focusing inside a material. Then, the "real" beam spot size was probably bigger than that, but it was still within the same size order of magnitude.) Given the thermal diffusivity of Yb:YAG $\left(3 \times 10^{-6} \mathrm{~m}^{2} / \mathrm{s}\right)$ [36], and according to expression (1), the critical frequency $\left(f_{c r}\right)$ is of about $280 \mathrm{kHz}$, yielding a normalized frequency $f_{n} \approx 1.75$, i.e. basically the critical frequency for thermal accumulation. At this frequency thermal annealing effects are activated while keeping the waveguide's morphology, that is, avoiding the drastic morphological changes which would be observed if waveguides were fabricated at the highest repetition rates (i.e around the $\mathrm{MHz}$ range) [15]. Structures were written by translating the sample at $2.5 \mathrm{~mm} / \mathrm{s}$. The pulse energy was set to $600 \mathrm{~nJ}$ yielding a pulse fluence of $\sim 7 \mathrm{~J} / \mathrm{cm}^{2}$.

(b) Non-thermal regime: An amplified Ti:sapphire laser system providing $120 \mathrm{fs}$ pulses at $\lambda_{0}=796 \mathrm{~nm}$ and $1 \mathrm{kHz}$ of repetition rate. For all experiments, the laser polarization was again adjusted to be perpendicular to both the incident beam and sample translation direction. The laser beam was focused with a $20 \times$ microscope objective (numerical aperture, $\mathrm{NA}=0.4$ ), giving a spot size of $2.5 \mu \mathrm{m}$. In this case the normalized frequency is about $f_{n} \approx 0.002$, which means that thermal accumulation between subsequent pulses is almost negligible. The sample was translated at $4 \mu \mathrm{m} / \mathrm{s}$ so that pulse overlapping was also kept constant with respect to the $500 \mathrm{kHz}$ waveguides. Two different on-target energies were used: $400 \mathrm{~nJ}$ and $600 \mathrm{~nJ}$. The use of these two pulse energies allows for comparative studies with the $500 \mathrm{kHz}$ structure maintaining fixed the pulse energy $(600 \mathrm{~nJ})$, or the pulse fluence $\left(\sim 8 \mathrm{~J} / \mathrm{cm}^{2}\right)$, respectively.

For both regimes described, the Gaussian focal spot size was always calculated using the NA, beam diameter and wavelength of the lasers. Both laser set ups are being extensively used in a variety of everyday experiments and therefore they are very well characterized. Furthermore, any slight variation from the calculated value could never produce the strong thermal effects that are investigated in the present work both by micro-spectroscopy and optical propagation experiments 


\subsection{Waveguide's propagation properties}

The waveguiding properties of the resulting structures as optical waveguides were firstly investigated by end-face coupling experiments using a linearly polarized He-Ne laser (at wavelength of $632.8 \mathrm{~nm}$ ). For this purpose, laser radiation was focused into the waveguide using a $20 \times$ microscope objective and the laser light propagating along the waveguide was collected using another $20 \times$ microscope objective. The obtained near-field intensity distribution of the fundamental mode was recorded with a charge coupled device (CCD) camera. The propagation losses of the $\mathrm{WG}$ were determined by using the Fabry-Pérot method at 632.8 nm [37].

\subsection{Micro-Photoluminescence and Raman characterization}

For $\mu$-PL experiments studies of the waveguide's crosssection, an Olympus BX-41 fibre-coupled confocal microscope was used. The continuous wave $920 \mathrm{~nm}$ radiation from a Ti:sapphire laser was focused $5 \mu \mathrm{m}$ deep below the sample surface using a $50 \times$ microscope objective with numerical aperture NA $=0.6$. The theoretical lateral and axial resolution of the confocal system were estimated to be close to $1 \mu \mathrm{m}$. In this configuration, the $920 \mathrm{~nm}$ laser radiation locally excites the $\mathrm{Yb}^{3+}$ ions from their fundamental state ${ }^{2} \mathrm{~F}_{7 / 2}$ up to the upper level of the ${ }^{2} \mathrm{~F}_{5 / 2}$ manifold [34]. The subsequent ${ }^{2} \mathrm{~F}_{5 / 2} \rightarrow{ }^{2} \mathrm{~F}_{7 / 2}$ emissions from the $\mathrm{Yb}^{3+}$ ions is collected using the same microscope objective and, after passing thorough a confocal aperture, is analyzed by a CCD detector attached to a fibre-coupled spectrometer (SPEX500M). The Yb:cYAG sample was mounted on an XY motorized stage with a spatial resolution of $100 \mathrm{~nm}$. The motorized stage and CCD were controlled with LabSpec(Csoftware for automatic acquisition and analysis of the obtained spectral data. Three-dimensional maps of the $\mathrm{Yb}^{3+}$ spectral properties (i.e., emitted intensity, emission bandwidth, and energy position of the emission lines) were obtained by fitting Lorentzian line-shapes to the spectra and by plotting the values obtained for each measuring point. The plot and graphical analysis of the obtained results was performed with the WSMPCsoftware [38].

For $\mu$-Raman measurements a Renishaw inVia Reflex confocal microscope was used. Raman spectra were measured using the $514 \mathrm{~nm}$ line of an Argon laser. Power on the sample was of $10 \mathrm{~mW}$, and a $50 \times, 0.75$ NA objective was used to focus $5 \mu \mathrm{m}$ below the sample surface. Pre-calibration of the equipment was always routinely performed on a crystalline Silicon reference. Phonon modes were assigned following prior work by Papagelis et al. [39].

\section{Results and discussion}

\subsection{Waveguiding properties}

Figure 1 (top row) shows the optical microscope crosssection views of the modification tracks formed with 1 and $500 \mathrm{kHz}$ pulse repetition rates. Clear differences in the changes can be observed in the morphology of the structures fabricated using the 1 and $500 \mathrm{kHz}$ systems. Firstly, a weaker contrast and a smoother modification is noted for the structure fabricated in the thermal regime $(500 \mathrm{kHz})$, suggesting not only a lower density of defects, but also less intense stress profiles as a result of the thermal process. Similar effects have been already reported in lithium niobate waveguides, and have been attributed to the presence of heat accumulation and rapid thermal annealing [15]. In addition, previous studies on post-fabrication annealing of these types of waveguides in Nd:cYAG samples showed that only annealing temperatures of above $1000^{\circ} \mathrm{C}$ could significantly reduce both the density of defects and the induced stress fields [30].

The bottom row in Fig. 1 shows the corresponding mode profiles recorded at $632.8 \mathrm{~nm}$ for each structure. The propagation modes included in Fig. 1 were obtained with the laser polarization parallel to the damage tracks. Waveguiding was also observed in both structures for the orthogonal polarization (electric field perpendicular to the damage tracks) but with a lower coupling efficiency and larger propagation looses. The waveguide fabricated using $500 \mathrm{kHz}$ repetition rate displayed a smoother, more symmetric and more extended propagation mode. This is in contrast to the propagation modes observed for the waveguides fabricated in the non-thermal regime $(1 \mathrm{kHz})$, which seem to be fully confined between the damage tracks. Thus, the propagation modes unequivocally suggest that thermal accumulation modifies the refractive index map, smoothing it and reducing the refractive index contrast.

Finally, we measured the Fabry-Pérot interference patterns for each structure at $632.8 \mathrm{~nm}$ (not shown for the sake of brevity). The obtained propagation losses have been indicated in Fig. 1. As can be observed, the presence of heat accumulation and rapid annealing processes have strongly reduced the propagation losses from $\sim 2 \mathrm{~dB} / \mathrm{cm}$ down to $1 \mathrm{~dB} / \mathrm{cm}$. Thus, it can be concluded that, at least in the case of polycrystalline ceramics, the presence of thermal accumulation process during fabrication can result in a strong improvement of the waveguide propagation properties. It is important to note that the fabrication parameters here reported are only intended to demonstrate how the heat accumulation effects associated with the laser writing technique in crystals can be used to substantially improve the device quality. A further development of the waveguide quality can therefore be expected following complete optimization of the process presented here. 
Fig. 1 (Top) Optical

microscope cross-section views

of the modification tracks

formed with 1 and $500 \mathrm{kHz}$

pulse repetition rates. (Bottom)

Corresponding mode profiles

recorded at $632.8 \mathrm{~nm}$ for each

structure. The propagation

losses obtained in each case are indicated. Common scale bar is $20 \mu \mathrm{m}$

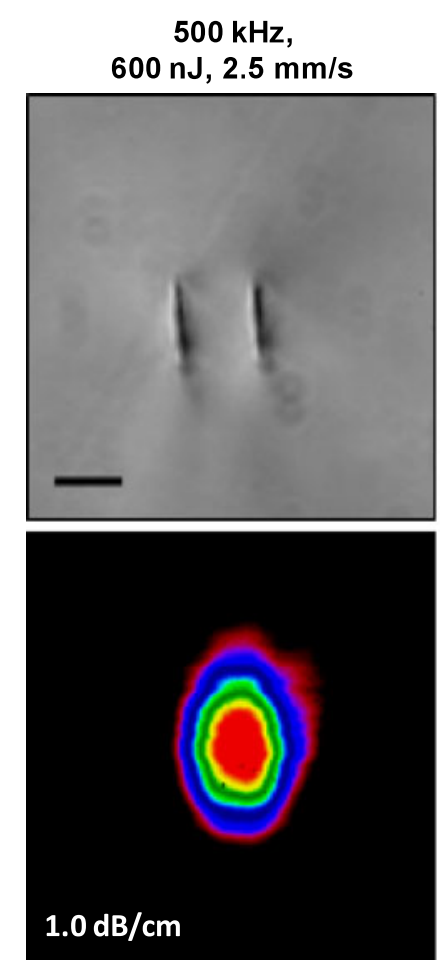

$500 \mathrm{kHz}$,

$600 \mathrm{~nJ}, 2.5 \mathrm{~mm} / \mathrm{s}$

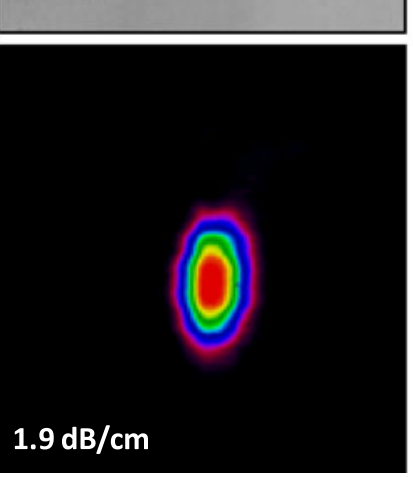

$1 \mathrm{kHz}$, $400 \mathrm{~nJ}, 4 \mathrm{\mu m} / \mathrm{s}$

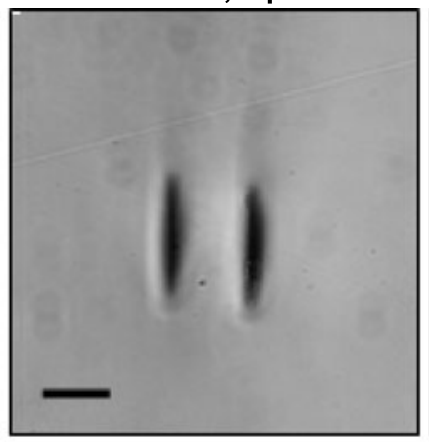

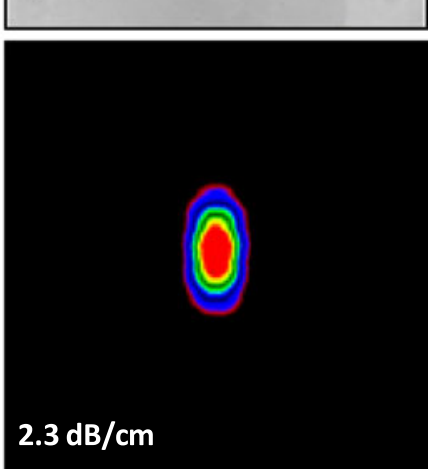

\subsection{Fluorescence properties}

The different propagation properties observed suggest that the presence of thermal accumulation leads to different modification of the YAG ceramic network and hence, to different refractive index maps. In order to obtain a further understanding of the microstructural modifications induced by the DLW process, the $\mathrm{Yb}^{3+}$ based fluorescence maps of the different structures have been measured.

Figure 2 presents a typical $\mu$-PL emission spectrum obtained using our confocal microscope. It consists of a narrow and intense peak (at $\approx 10300 \mathrm{~cm}^{-1}$ ) corresponding to the $\mathrm{Yb}^{3+}$ zero-phonon transition and a broad laser emission band (at $\approx 9700 \mathrm{~cm}^{-1}$ ) attributed to radiative transitions from the lowest energy sublevel of the ${ }^{2} \mathrm{~F}_{5 / 2}$ manifold to intermediate sublevels of the ${ }^{2} \mathrm{~F}_{7 / 2}$ ground state [34]. Between these two bands a rich structure is observed. This is conventionally related to other inter Stark transitions as well as to phonon side-bands [34]. The bottom graph in Fig. 2 shows the detail of the $\mu$-PL emission spectra obtained when the $920 \mathrm{~nm}$ excitation spot was localized at an unmodified volume (bulk), within the waveguide's volume (i.e. between damage tracks) and at one of the damage tracks. The data correspond to the waveguide written at $1 \mathrm{kHz}$ and with a pulse energy of $600 \mathrm{~nJ}$. It is clear from these detailed spectra that the $\mathrm{Yb}^{3+}$ emission spectrum has been strongly modified by the DLW process. The emitted intensity at the zerophonon line has been reduced at damage tracks. This reduction is accompanied by an associated increase in the emitted intensity at $10200 \mathrm{~cm}^{-1}$. We attribute this behaviour to the presence of a high density of defects as a consequence of the optical breakdown induced at focal volume. These defects lead to an increment in the effective photon path due to multiple scattering. In other words, scattering induces a random trajectory in the fluorescence photons. In the case of $\mathrm{Yb}^{3+}$ doped materials, changes in the effective photon path modify the relative intensities of the different emission lines due to the so-called self-trapping (or self-absorption) of emission [40, 41]. For those fluorescence lines overlapping with absorption transitions, any increment in the photon path between the excitation volume and the detection system, leads to an intensity reduction (since self-absorption is enhanced). This is the case for the zero phonon line. This reduction in the fluorescence intensity of self-absorbed emission lines in turns causes an intensification of those emission bands not affected by self-absorption (as observed for the $10250 \mathrm{~cm}^{-1}$ emission band). According to this argument, the change in the ratio between the emitted intensities at 10250 and $10325 \mathrm{~cm}^{-1}$ is a direct indicator of the presence of defects. Figure 3 shows the spatial variation of the ratio between the emitted intensities at 10250 and $10325 \mathrm{~cm}^{-1}$ in the three waveguides under study in this work. In the three cases this ratio is only modified at the damage tracks. This fact clearly indicates that in each case, defects have been created only in damage tracks, and the waveguide's volume being almost free of them. The magnitude of the observed change has been found to be strongly dependent on the repetition rate used during waveguide writing while the pulse energy 
was not so critical. For the waveguide written with $500 \mathrm{kHz}$ pulses the induced ratio change has been found to be not more than $200 \%$ (from an "intensity ratio" value of 0.1 at the bulk to an "intensity ratio" value around 0.2 at damage tracks), whereas for the waveguides written with $1 \mathrm{kHz}$ pulses this change has been found to be close to $800 \%$ (from

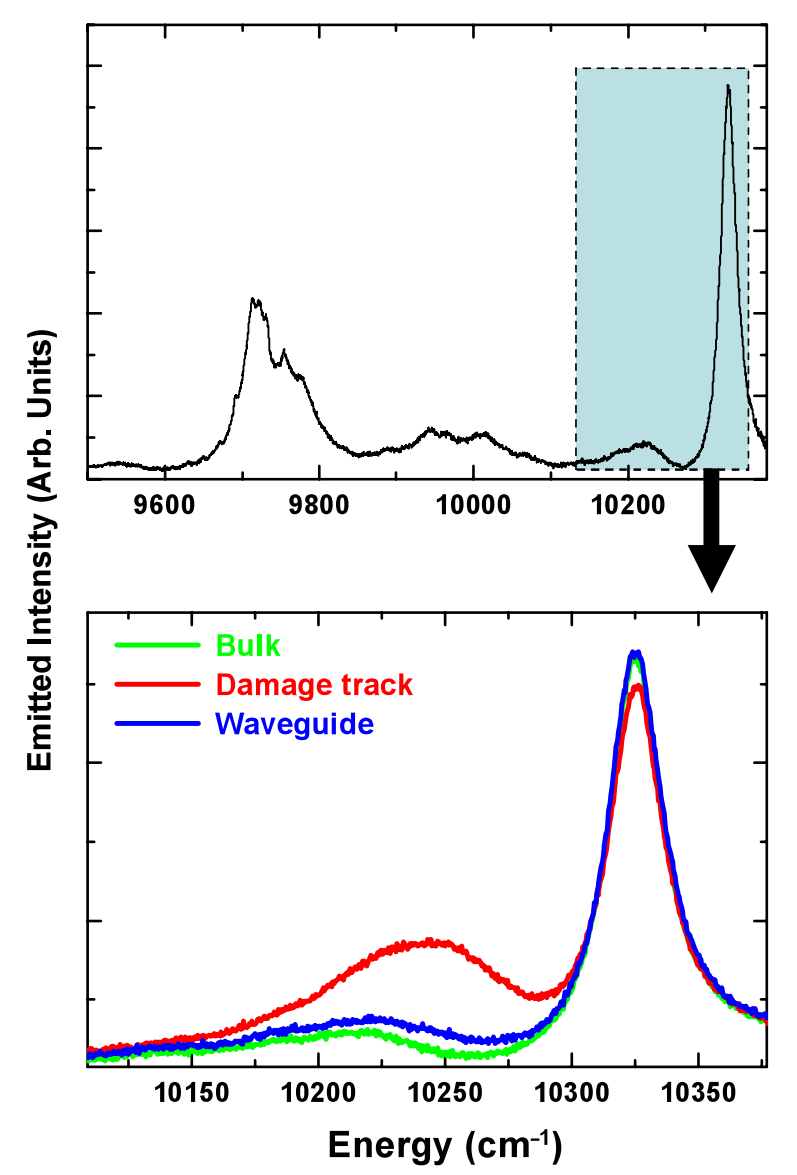

Fig. 2 (Top) Typical $\mu$-PL emission spectrum obtained from our confocal microscope. (Bottom) Detail of the $\mu$-PL emission spectra obtained when the $920 \mathrm{~nm}$ excitation spot was localized at an unperturbed volume (bulk), at the waveguide's volume (i.e. between damage tracks) and at one of the damage tracks. Data correspond to the waveguide written at $1 \mathrm{kHz}$ and with a pulse energy of $600 \mathrm{~nJ}$ an "intensity ratio" value of 0.1 at the bulk to an "intensity ratio" value slightly below 0.8 at damage tracks). Since, as discussed before, this fluorescence ratio is indicative of the presence of defects, the data presented in Fig. 3 unequivocally indicate that the presence of heat accumulation leads to a partial recombination of laser-induced defects. This is, indeed, in agreement with the lower propagation losses obtained for these waveguides and is also in accordance with previous studies that reveal that when fs pulses are focused inside crystalline materials, two different kind of defects are created at focus; permanent defects (associated to irreversible optical breakdown of the crystalline network) and thermally removable defects [42]. We postulate that the heat accumulation has led to the partial recombination of thermally removable defects in the $500 \mathrm{kHz}$ written waveguides. In the waveguides written with $1 \mathrm{kHz}$ the density of defects is larger at damage tracks, since both permanent and thermally removable defects are present [42].

The lower density of defects in the $500 \mathrm{kHz}$ waveguide also explains the more symmetrical propagation mode obtained for this structure (see Fig. 1). It is known that in double-line waveguides, the mode confinement (mode size) in the dimension perpendicular to damage tracks is determined by the refractive index barrier (reduction) caused by the lattice damage at damage tracks [30]. Thus, in the thermal waveguides, this damage is strongly reduced, lowering the refractive index barrier and thus reducing the horizontal confinement.

Further information about the microstructural changes induced in the YAG network can be obtained from the analysis of the spectral position of emission bands. It is known that the spectral positions of fluorescence lines of rare-earth ions in YAG (as in any other crystal) are strongly dependent of the crystal field (CF) acting on them [34]. Since the CF is determined by the inter-atomic distances between the luminescent ions and the closest neighbours, any modification in these distances causes a variation in the $\mathrm{CF}$ and consequently, in the spectral position of emission lines. In order to take advantage of this fact we have measured the spatial variation of the spectral position of the zero-phonon line in
Fig. 3 Spatial variation of the ratio between the ytterbium emitted intensities at 10250 and $10325 \mathrm{~cm}^{-1}$ in the three waveguides under study in this work. Scale bar is $20 \mu \mathrm{m}$
$500 \mathrm{kHz}$, $600 \mathrm{~nJ}, 2.5 \mathrm{~mm} / \mathrm{s}$

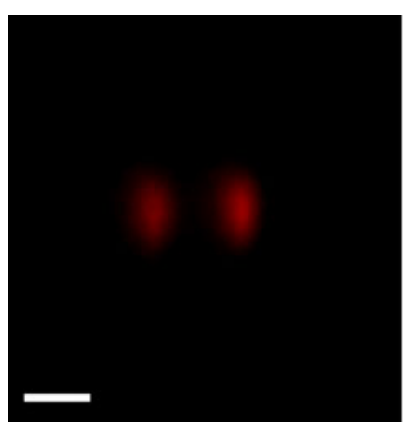

$1 \mathrm{kHz}$,

$400 \mathrm{~nJ}, 4 \mu \mathrm{m} / \mathrm{s}$

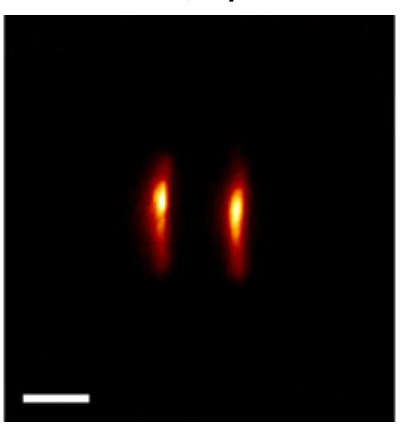

$1 \mathrm{kHz}$, $600 \mathrm{~nJ}, 4 \mu \mathrm{m} / \mathrm{s}$

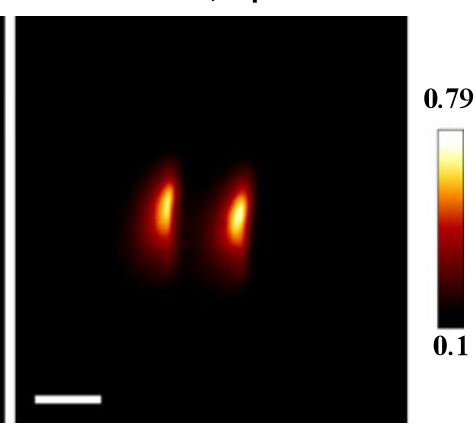


Fig. 4 Spatial variation of the spectral position of the ytterbium zero-phonon line in the three waveguides under study. Scale bar is $20 \mu \mathrm{m}$

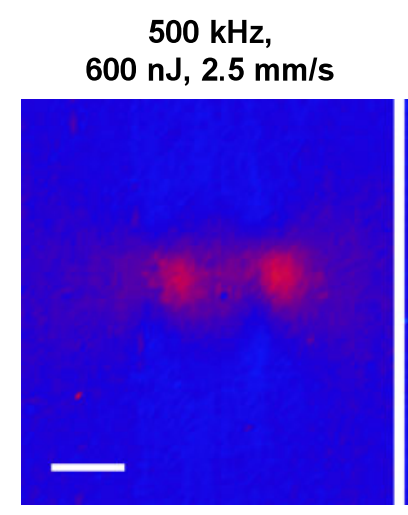

$500 \mathrm{kHz}$, $400 \mathrm{~nJ}, 4 \mathrm{\mu m} / \mathrm{s}$

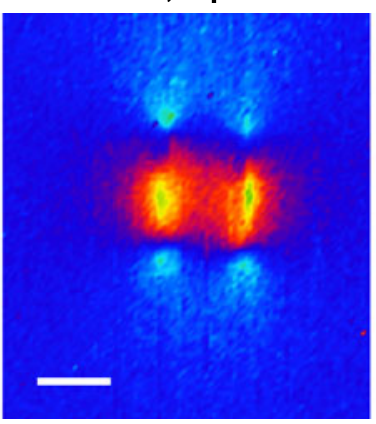

$1 \mathrm{kHz}$, $600 \mathrm{~nJ}, 4 \mu \mathrm{m} / \mathrm{s}$

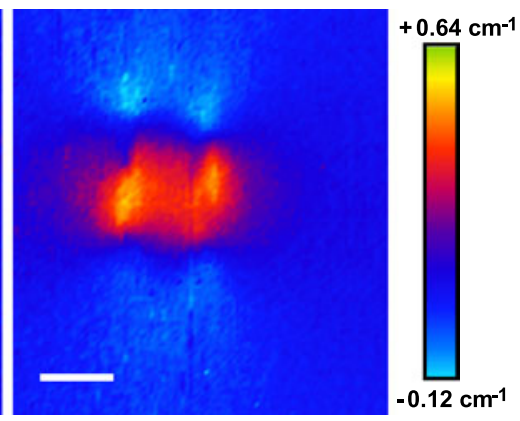

the three waveguides under study, with our results shown in Fig. 4. It is clear that the zero-phonon line is shifted to higher energies at damage tracks and between them and to lower energies at their apexes. According to previous works on Nd:YAG ceramics [30], these shifts can be attributed to the appearance of a compressive stress (causing a reduction in the inter-atomic distances) at damage tracks and between them. This is accompanied by a tensile stress at the apexes of damage tracks. Note that in the case of $\mathrm{Nd}$ :cYAG compressive stress was traced by the red-shift of emission bands whereas in the case of Yb:cYAG it causes a blue-shift of the zero-phonon line [30]. This fact suggests that the pressure induced spectral shift of $\mathrm{Nd}^{3+}$ and $\mathrm{Yb}^{3+}$ fluorescence lines is the opposite. Unfortunately, there are not reports on the behaviour of $\mathrm{Yb}^{3+}$ ions in compressed YAG system, so this hypothesis cannot be supported at this stage.

The comparison between the fluorescence images of Fig. 4 allows us to obtain a first order comparison between the extension and magnitude of the stress maps generated in the presence and absence of heat accumulation. The fluorescence image of the $500 \mathrm{kHz}$ waveguide has been found to be of lower contrast than those corresponding to the $1 \mathrm{kHz}$ waveguides. This suggests that heat accumulation has not only produced defect recombination but also partial annealing of the strain field (which indeed is expected to be activated for temperatures above $800^{\circ} \mathrm{C}$ ) [30]. Since, in a first order approximation, the stress-induced refractive index change is proportional to the induced fluorescence shift, the data of Fig. 4 imply a lower refractive index modulation for the waveguides written in the thermal regime. This is indeed confirmed by the larger waveguide propagation mode obtained for these waveguides (see Fig. 1).

As a secondary issue related with the data plotted in Fig. 4, it is necessary to point out the comparison between shift maps from two different waveguides (400 and $600 \mathrm{~nJ}$ pulse energy) made with the same repetition rate $(1 \mathrm{kHz})$. When we were increasing the fluence, the residual stress instead of increase suffered a slight decrease. As a matter of fact, the relation between irradiation fluence and induced stress not always follows a linear trend. Indeed, some previous works reported in other materials (M.S. Amer et al. [43]), have established that a "saturation" point on the material's structure reaction to the pulse fluence increment. So, for pulse fluencies above this value, the induced stress reduces its value with the increasing pulse fluence. In addition, such type of trend, which includes a saturation point, seems to be more probable when writing laser beams linearly polarized are used (as in our case) [43]. The relationship and quantitative trend between pulse energy deposited (irradiation fluence) on material's lattice, and the induced stress on that lattice does not seem to be trivial, and it deserves a more specific investigation to be done in the future.

\subsection{Raman measurements}

To obtain a deeper understanding of the induced microstructural changes and also to corroborate previous assumptions that ytterbium blue-shift is related to an effective local compression, $\mu$-Raman spectroscopy of the three waveguide has been analyzed. Figure 5(top) shows the Raman spectra as obtained from the damage tracks, the apexes, the waveguide, and the bulk non irradiated volume. It is clear that both the intensity and position of Raman modes depends on the excited volume. Indeed, the Raman modes shift to higher vibration energies at both the damage tracks and within the waveguide volume, whereas they shift to lower vibration energies at apexes. This fact is most clearly appreciated in the Raman images shown at the bottom of Fig. 5, in which we have displayed the $2 \mathrm{D}$ map of the induced shift in the $A_{1 g}$ Raman mode at $371 \mathrm{~cm}^{-1}$. It is important to note that the same behaviour was obtained for all the Raman modes, which are not shown here for the sake of brevity. Based on the work of J. Arvanitidis et al. [44], who measured the pressure dependence of all phonon modes, we have concluded that a permanent compressive stress has been created at damage tracks and between them, these being compensated by the appearance of a tensile stress at the apexes (as assumed in Sect. 3.2). The stress maps that can be inferred 
Fig. 5 (Top) Raman spectra as obtained from the damage tracks, the apexes, the waveguide, and the bulk. (Bottom) Raman images showing the 2D map of the induced shift in the $\mathrm{A}_{1 \mathrm{~g}}$ Raman mode at $371 \mathrm{~cm}^{-1}$. Scale bar is $20 \mu \mathrm{m}$

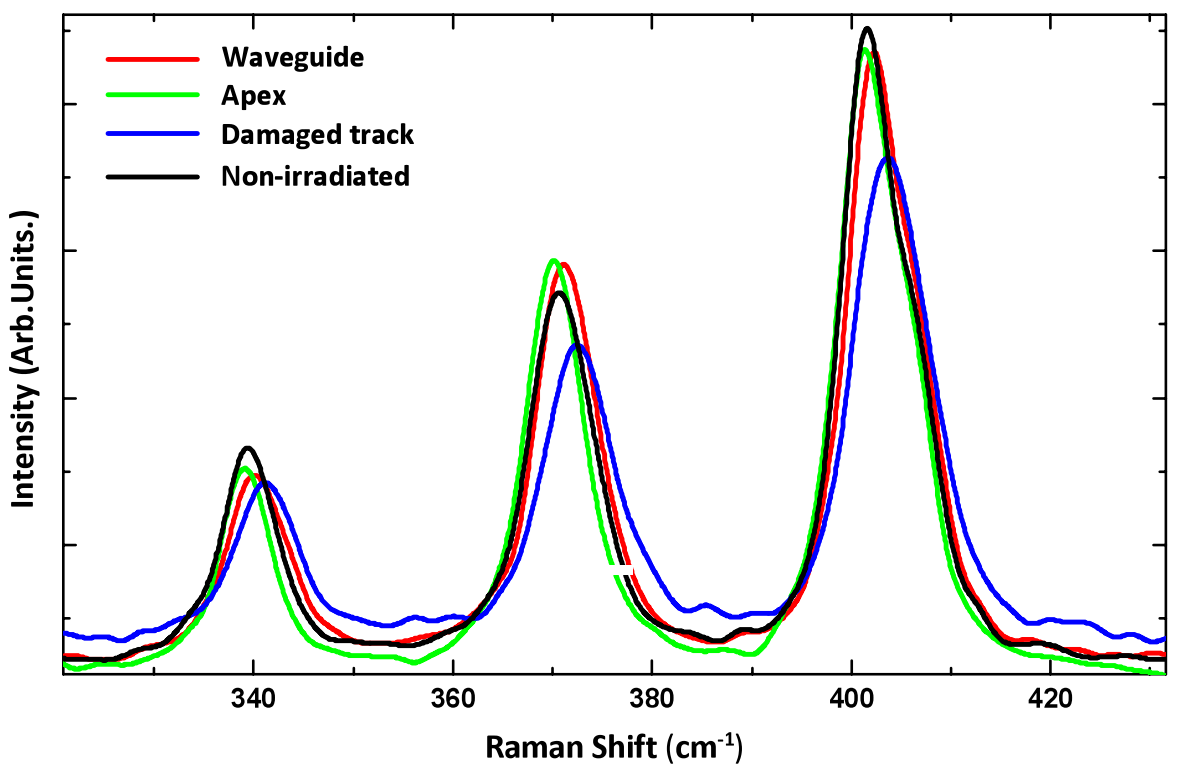

$500 \mathrm{kHz}$, $600 \mathrm{~nJ}, 2.5 \mathrm{~mm} / \mathrm{s}$

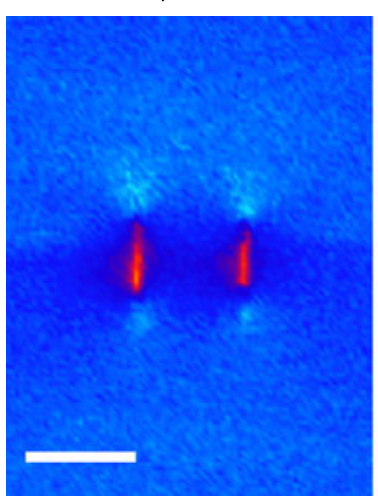

$1 \mathrm{kHz}$, $400 \mathrm{~nJ}, 4 \mu \mathrm{m} / \mathrm{s}$

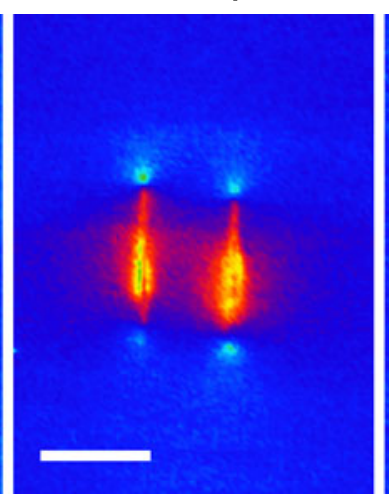
$600 \mathrm{~nJ}, 4 \mu \mathrm{m} / \mathrm{s}$

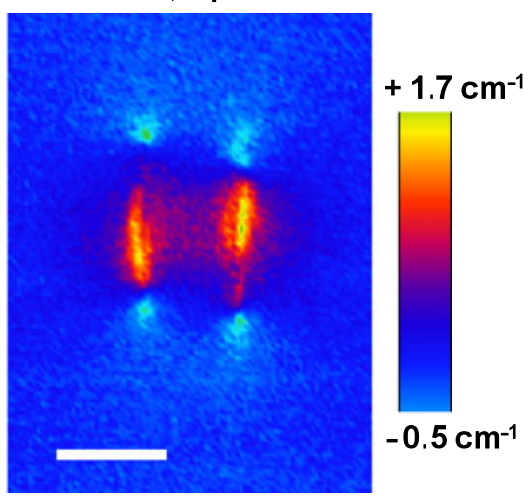

from the Raman images are in accordance with those previously obtained for DLW waveguides in Nd:cYAG [30].

The previous work of J. Arvanitidis et al. reported the experimental Grüneisen parameters of all the observed phonon modes [44]. These data allow us to estimate the magnitude of the stress fields associated with each Raman mode, which yield very similar values, as expected for almost hydrostatic conditions. Therefore, average values for the stress fields were obtained by using the different Grüneisen parameters of each phonon mode. The maximum residual compressive stress has been found at the damage tracks, being about $\sim 0.3$ and $0.5 \mathrm{GPa}$ for the waveguides written in the thermal and non-thermal regime, respectively. Thus, we have confirmed that rapid annealing (present in $500 \mathrm{kHz}$ waveguides) has partially removed the residual stress created by DLW. This is consistent with the low contrast obtained in the fluorescence images of these waveguides (see Fig. 4). Finally, the maximum residual stress for $1 \mathrm{kHz}$ waveguides is comparable to that obtained in Nd:cYAG waveguides written with similar pulse energies [30].

\section{Conclusions}

In summary, optical waveguides in Yb:YAG ceramics have been fabricated by DLW. The effect that rapid annealing has on the propagation features and structural properties of the obtained waveguides has been investigated by using laser repetition rates well above and well below the critical frequency. It has been found that those waveguides written in the thermal regime, i.e. in presence of rapid annealing shows better propagation properties (more symmetrical mode and lower propagation losses) than those written in the nonthermal regime. These improved properties have been successfully explained in terms of a partial recombination of defects, which has been monitored through the analysis of the specific features of ytterbium emissions (ratio between selfabsorbed and non self-absorbed ytterbium bands). In addition, Raman and fluorescence images both concluded that heat accumulation also lead to a reduction in the residual stress generated in the irradiated areas. 
The remarkable laser properties of ytterbium ions in YAG ceramics together with the improved propagation properties of the waveguides written in the presence of heat accumulation effects, make the structures reported in this work promising candidates for efficient integrated laser sources.

Acknowledgements This work has been supported by the Spanish Ministerio de Ciencia y Tecnología (MAT2007-64686, MAT200906580-E/MAT, FIS2009-09522 and CSD2007-00013), the Consejería de Educación de la Comunidad de Madrid, Universidad Autónoma de Madrid (UAM-CAM) (CCG08-UAM/MAT-4434, PHAMA P2009/ MAT1756), CEAL-Banco Santander, the Brazilian CAPES and CNPq, by Chinese NSFC (10925524), the Spanish Ministerio de Ciencia and Argentinean CONICET (PIP 11220090100394), and the UK Engineering and Physical Sciences Research Council (EPSRC), grant numbers EP/G030227/1 and EP/D047269/1. We would also like to thank Renishaw for the long-term loan of an inVia Reflex Raman microscope as part of the Renishaw-Heriot-Watt Strategic Alliance.

\section{References}

1. D. Du, X. Liu, G. Korn, J. Squier, G. Mourou, Appl. Phys. Lett. 64, 3071 (1994)

2. K.M. Davis, K. Miura, N. Sugimoto, K. Hirao, Opt. Lett. 21, 1729 (1996)

3. Y. Kawata, H. Ueki, Y. Hashimoto, S. Kawata, Appl. Opt. 34, 4105-4110 (1995)

4. E.N. Glezer, M. Milosavljevic, L. Huang, R.J. Finlay, T.-H. Her, J.P. Callan, E. Mazur, Opt. Lett. 21, 2023 (1996)

5. Y. Sikorski, A.A. Said, P. Bado, R. Maynard, C. Florea, K.A. Winick, Electron. Lett. 36, 226-227 (2000)

6. J. Burghoff, C. Grebing, S. Nolte, A. Tunnermann, Appl. Phys. Lett. 89, 8 (2006)

7. W. Watanabe, T. Asano, K. Yamada, K. Itoh, Opt. Lett. 28, 2491 (2003)

8. N. Takeshima, Y. Narita, S. Tanaka, Y. Kuroiwa, K. Hirao, Opt. Lett. 30, 352 (2005)

9. Y. Chen, K. Sugioka, K. Midorikawa, Opt. Lett. 29, 2007 (2004)

10. G. Zhou, M. Gu, Opt. Lett. 31(18), 2783 (2006)

11. J. Burghoff, S. Nolte, A. Tünnermann, Appl. Phys. B 89, 127 (2007)

12. M. Hughes, W. Yang, D. Hewak, Appl. Phys. Lett. 90, 131113 (2007)

13. A.M. Streltsov, N.F. Borrelli, J. Opt. Soc. Am. B 19, 2496 (2002)

14. N.T. Nguyen, A. Saliminia, S.L. Chin, R. Vallée, Appl. Phys. B 85, 145 (2006)

15. A.H. Nejadmalayeri, P.R. Herman, Opt. Express 15, 10842 (2007)

16. R. Osellame, M. Lobino, N. Chiodo, M. Marangoni, G. Cerullo, R. Ramponi, H.T. Bookey, R.R. Thomson, N.D. Psaila, A.K. Kar, Appl. Phys. Lett. 90, 241107 (2007)

17. H.T. Bookey, R.R. Thomson, N.D. Psaila, A.K. Kar, N. Chiodo, R. Osellame, G. Cerullo, IEEE Photonics Technol. Lett. 19, 892 (2007)
18. W.F. Silva, C. Jacinto, A. Benayas, J.R. Vazquez de Aldana, G.A. Torchia, F. Chen, Y. Tan, D. Jaque, Opt. Lett. 35, 916-918 (2010)

19. S.M. Eaton, C.A. Merchant, R. Iyer, A.J. Zilkie, A.S. Helmy, J.S. Aitchison, P.R. Herman, D. Kraemer, R.J.D. Miller, C. Hnatovsky, R.S. Taylor, Appl. Phys. Lett. 92, 081105 (2008)

20. G.A. Torchia, A. Rodenas, A. Benayas, E. Cantelar, L. Roso, D. Jaque, Appl. Phys. Lett. 92, 111103 (2008)

21. B. McMillen, K.P. Chen, H. An, S. Fleming, V. Hartwell, D. Snoke, Appl. Phys. Lett. 93, 111106 (2008)

22. C.B. Schaffer, J.F. Garcia, E. Mazur, Appl. Phys. A 76, 351-354 (2003)

23. C.B. Schaffer, A. Brodeur, J.F. Garcia, E. Mazur, Opt. Lett. 26, 93 (2001)

24. S. Juodkazis, H. Misawa, I. Maksimov, Appl. Phys. Lett. 85, 5239 (2004)

25. S.M. Eaton, H. Zhang, P.R. Herman, F. Yoshino, L. Shah, J. Bovatsek, A.Y. Arai, Opt. Express 13, 4708 (2005)

26. A.A. Kaminskii, Laser Photonics Rev. 1, 93 (2007)

27. A. Ródenas, G. Zhou, D. Jaque, Gu. Min, Appl. Phys. Lett. 93, 151104 (2008)

28. A.G. Okhrimchuk, A.V. Shestakov, I. Khrushchev, J. Mitchell, Opt. Lett. 30, 2248 (2005)

29. J. Siebenmorgen, K. Petermann, G. Huber, K. Rademaker, S. Nolte, A. Tünnermann, Appl. Phys. B 97, 251 (2009)

30. A. Ródenas, G.A. Torchia, G. Lifante, E. Cantelar, J. Lamela, F. Jaque, L. Roso, D. Jaque, Appl. Phys. B 95, 85 (2009)

31. A. Benayas, W.F. Silva, C. Jacinto, E. Cantelar, J. Lamela, F. Jaque, J.R. Vázquez de Aldana, G.A. Torchia, L. Roso, A.A. Kaminskii, D. Jaque, Opt. Lett. 35, 330 (2010)

32. J. Dong, A. Shirakawa, K. Ueda, H. Yagi, T. Yanagitani, A.A. Kaminskii, Opt. Lett. 32, 1890 (2007)

33. H. Yoshioka, S. Nakamura, T. Ogawa, S. Wada, Opt. Express 17, 8919 (2009)

34. B. Henderson, G.F. Imbusch, Optical Spectroscopy of Inorganic Solids (Oxford University Press, New York, 1989)

35. J. Siebenmorgen, T. Calmano, K. Petermann, G. Huber, Opt. Express 18, 16036 (2010)

36. T. Taira, IEEE J. Sel. Top. Quantum Electron. 13, 798 (2007)

37. R. Regener, W. Sohler, Appl. Phys. B 36, 143 (1985)

38. I. Horcas, R. Fernandez, J.M. Gomez-Rodriguez, J. Colchero, J. Gomez-Herrero, A.M. Baro, Rev. Sci. Instrum. 78, 013705 (2007)

39. K. Papagelis, G. Kanellis, S. Ves, G.A. Kourouklis, Phys. Status Solidi, B Basic Res. 233, 134 (2002)

40. D.S. Sumida, T.Y. Fan, Opt. Lett. 19, 1343 (1994)

41. F. Auzel, G. Baldacchini, L. Laversenne, G. Boulon, Opt. Mater. 24, 103 (2003)

42. A. Benayas, D. Jaque, Ben McMillen, K.P. Chen, J. Appl. Phys. 107, 033522 (2010)

43. M.S. Amer, M.A. El-Ashry, L.R. Dosser, K.E. Hix, J.F. Maguire, Bryan Irwin, Appl. Surf. Sci. 242, 162-167 (2005)

44. J. Arvanitidis, K. Papagelis, D. Christofilos, H. Kimura, G.A. Kourouklis, S. Ves, Phys. Status Solidi, B Basic Res. 241, 31493154 (2004) 